\title{
Detection of a relic X-ray jet in Cygnus A
}

\author{
K. C. Steenbrugge, K. M. Blundell \\ University of Oxford, \\ Oxford, OX1 3JP, UK \\ E-mail: kcs@astro.ox.ac.uk; kmb@astro.ox.ac.uk \\ P. Duffy \\ School of Mathermatics, UCD, \\ Dublin, 4, Ireland \\ E-mail: peter.duffy@ucd.ie
}

\begin{abstract}
We present a 200 ks Chandra ACIS-I image of Cygnus A, and discuss a long linear feature seen in its counterlobe. This feature has a non-thermal spectrum and lies on the line connecting the brighter hotspot on the approaching side and the nucleus. We therefore conclude that this feature is (or was) a jet. However, the outer part of this X-ray jet does not trace the current counterjet observed in radio. No X-ray counterpart is observed on the jet side. Using light-travel time effects we conclude that this X-ray $50 \mathrm{kpc}$ linear feature is a relic jet that contains enough low-energy plasma $\left(\gamma \sim 10^{3}\right)$ to inverse-Compton scatter cosmic microwave background photons, producing emission in the X-rays.
\end{abstract}

Keywords: radio galaxy - individual: Cygnus A.

\section{Introduction}

The Cygnus A cluster and galaxy (3C 405) are one of the brightest sources in the X-ray sky and have therefore been studied with every major X-ray satellite. In this paper we take advantage of the high spatial resolution of the Chandra satellite to study the linear jet-like feature revealed in the X-ray image within the counterlobe. The spatial resolution of the ACIS camera onboard Chandra allows us to spatially resolve the jet, lobes and hotspots from the central AGN.

Detection of X-ray photons that arise from inverse Compton up-scattered Cosmic Microwave Background (ICCMB) photons mandate the presence of relativistic particles with Lorentz factors of order $10^{3} .{ }^{1}$ Such particles will have lower Lorentz factors than ambient synchrotron-emitting particles radiating at the typically-observed radio wavelengths, assuming the magnetic field strengths in the lobes of radio galaxies are $\mathrm{nT}$ in size or lower. Thus lower Lorentz factor particles can signal the presence of relic (that is previously, but no longer detectably synchrotron emitting) plasma. ${ }^{2,3}$

With the physical size of Cygnus A being $130 \mathrm{kpc}$, and a redshift of $0.05607,{ }^{4}$ (assuming a cosmology of $H_{0}=73 \mathrm{~km} \mathrm{~s}^{-1} \mathrm{Mpc}^{-1}$ and $\Omega_{\mathrm{M}}=0.3$ and $\left.\Omega_{\Lambda}=0.7\right)$ the light-travel time between opposite lobes exceeds $\cos \theta \times 4 \times 10^{5}$ years, where $\theta$ is the angle between the axis of the radio source and our line-of-sight. Since the light we observe from opposite lobes is received at the same telescope time, this means that an observer on Earth sees the nearer lobe at a more recent epoch than the further lobe, which is seen at an earlier time (hence less evolved) in the radio galaxy's history.

\section{Chandra Observations}

The details of the observations are listed in Table 1 . The first 2 observations used the ACIS-S (Advanced CCD Imaging Spectrometer) instrument and were only used in the spectral analysis. The remaining eight observations analysed in this paper used the ACIS-I instrument in the VFAINT mode, which gives a reduced background after processing. All the data were obtained from the Chandra public archive and reduced with the standard threads in CIAO 3.3 , which included the updated calibration database CALDB 3.2.2. The background region was chosen from a low count rate region on the CCD containing the image of Cygnus A.

We aligned the AGN core detected in the $2-$ $10 \mathrm{keV}$ band of the different observations using the reproject_aspect thread and then added the images using the merge_all command in CIAO. The resulting image is shown in Fig. 1. For extraction of the spectra from different regions we used the specex- 
tract command using the specific badpixel file for each observation. The quoted errors on the X-ray luminosity and photon index is for $\Delta \chi^{2}=2$, the RMS of the $\Delta \chi^{2}$ distribution. We used the $\operatorname{SPEX}^{5}$ package for fitting the spectra.

Table 1. The list of the 8 observations of Cygnus A used in this paper. Listed are the date of the observation and the filtered exposure time.

\begin{tabular}{|l|l|r|}
\hline & date & exposure (ks) \\
\hline 1 & 21052000 & 34.72 \\
2 & 26052000 & 10.17 \\
3 & 15022005 & 25.80 \\
4 & 16022005 & 51.09 \\
5 & 19022005 & 25.44 \\
6 & 21022005 & 6.96 \\
7 & 22022005 & 23.48 \\
8 & 23022005 & 23.05 \\
9 & 25022005 & 16.04 \\
10 & 07092005 & 29.65 \\
\hline
\end{tabular}

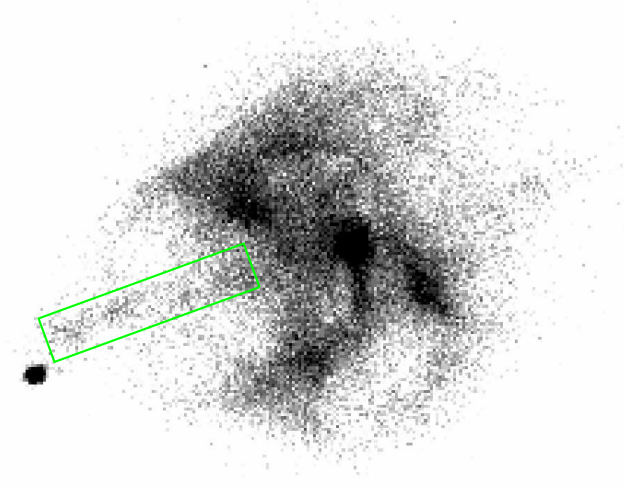

Fig. 1. The $0.2-10 \mathrm{keV}$ ACIS-I image clearly showing the linear counterjet-like feature delineated by the green box and the non-detection of anything corresponding to this on the jet side.

\section{Results}

A linear counterjet-like feature is easily detected in the $0.2-10 \mathrm{keV}$ image (see Fig. 1). The width of the counterjet is $\sim 5^{\prime \prime}$, and is resolved in our Chandra image. The width of the brightest knots observed in the 15 - GHz image are $\sim 2^{\prime \prime} .9{ }^{6}$ The X-ray counterjet feature is thus wider than its radio jets in Cygnus A. The green box in Fig. 1 indicates the region from which we extracted the spectrum for this feature. In- evitably, this box will contain some emission from the background thermal gas originating from the cluster in which Cygnus A is embedded. We rebinned the spectrum by a factor of 3 , and fitted the spectrum between $0.5-7 \mathrm{keV}$. The X-ray spectrum of the counterjet is well fitted $\left(\chi^{2}=1.1\right.$ for 1582 degrees of freedom) by a power-law with Galactic absorption ${ }^{7}$ of $3.5 \times 10^{25} \mathrm{~m}^{-2}$. The $2-10 \mathrm{keV}$ luminosity is $(7.0 \pm$ $0.12) \times 10^{35} \mathrm{~W}$ and the photon index is $1.70 \pm 0.02$ (see Fig. 2). The data are too poor to constrain the thermal component from the cluster.

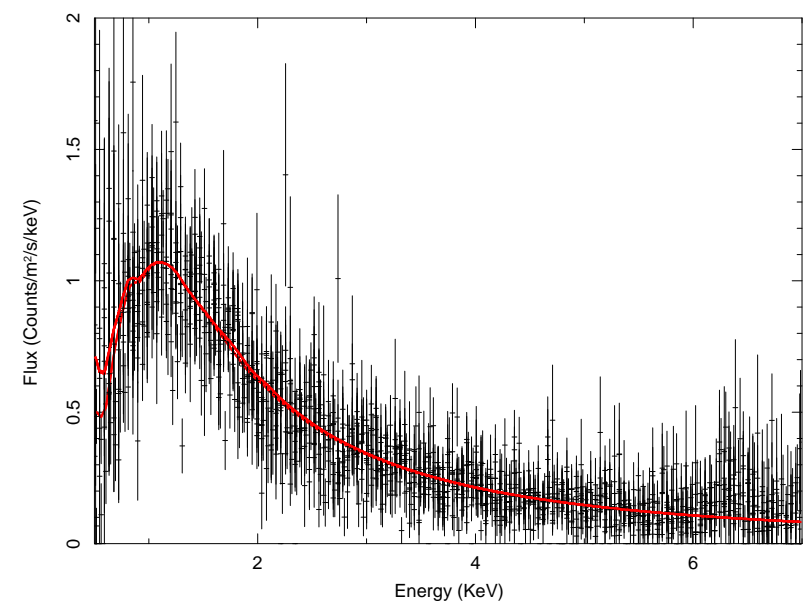

Fig. 2. The $0.5-7 \mathrm{keV}$ flux spectrum for the 10 different observations. The slight excess around $6.4 \mathrm{keV}$ is due to the thermal emission. The slight different fit and larger spread in data points is a result of the different calibration between ACIS-I and ACIS-S.

The brighter features in Cygnus A were studied ${ }^{8}$ before, but not the counterjet. This study convincingly showed that the higher temperature gas is at the outer edge, i.e. the contact discontinuity. The more centrally located gas is quite a bit cooler ranging between 3.80 and $4.28 \mathrm{keV}$ and can be explained as being due to the jet break-out phase. ${ }^{9,10}$ The location of the counterjet feature is inconsistent with it either belonging to the gas heated by the jet breakout or the contact discontinuity.

Fitting the counterjet feature with a thermal model gives a poorer fit, namely $\chi^{2}=1.2$ (for the same degrees of freedom), for a temperature of $6.3 \mathrm{keV}$ and an emission measure of $5.59 \pm$ $0.13 \times 10^{71} \mathrm{~m}^{-3}$. From this emission measure, using $Y=n_{\mathrm{e}} n_{\mathrm{H}} V$ we derive an electron density of $3.1 \times 10^{5} \mathrm{~m}^{-3}$. We assumed $n_{\mathrm{e}}=2.1 n_{\mathrm{H}}$ and a volume 
of $1.22 \times 10^{61} \mathrm{~m}^{3}$. The upper limit to the thermal electron density ${ }^{11}$ in the western lobe of Cygnus $\mathrm{A}$ is $4 \times 10^{2} \mathrm{~m}^{-3}$ for an isotropic random magnetic field; this could be higher by as much as two orders of magnitude if the magnetic field has many reversals. Even this extreme upper limit still falls short of the necessary electron density. We thus conclude that this feature is unlikely to be thermal.

The X-ray counterjet obeys the criteria $^{12}$ for jet idenitification: its length is more than four times larger than its width; it is separable at high spatial resolution from the surrounding features; and it is aligned with the compact core. More precisely, the counterjet lies on the same line that connects the brighter western hotspot (i.e. the brighter hotspot on the jet side) and the nucleus. Therefore, we conclude that the long linear feature identified on the east of the source is indeed related to jet activity and not part of the surrounding environment.

\subsection{Relic counterjet}

The inner part of the X-ray counterjet does overlay with the inner part of the counterjet detected in the radio images, however it does not make the $27^{\circ} 31^{\prime}$ bend observed in the $15-\mathrm{GHz}$ image; ${ }^{6}$ rather it extends along its original direction until just north of the bright eastern radio hotspot. There is a clear gap in emission between the end of the X-ray detected counterjet and the radio hotspot. We conclude that the X-ray detected counterjet is a relic for the following reasons: (i) it is extended transversely compared to the radio counterjet and radio jet, (ii) it does not overlay the outer radio counterjet, (iii) there is a gap in emission between the observed counterjet and the hotspots.

The inner part of the relic counterjet overlaps with the inner part of the current (radio) counterjet, and therefore we cannot constrain in this region the X-ray luminosity potentially coming from the inner current counterjet. However, for the outer X-ray counterjet there is a lack of associated $15-\mathrm{GHz}$ radio emission. ${ }^{6}$ This indicates a lack of high Lorentzfactor particles in this relic counterjet compared with those observed in active jets.

\section{Light-travel time effects and cooling}

The observation of X-ray emission from the counterjet side, and the absence of such emission from the approaching side of the source, places general constraints on the rate at which the X-ray emission must fade. Assuming symmetrical conditions, this depends purely on light-travel time arguments. We consider approaching and receding jets moving with speed $v=\beta_{\mathrm{HS}} c$ from the central source and making an angle $\theta$ with the observer's line of sight. When the approaching jet is observed to have extended out to a distance $d$, so that $t_{\text {app }}=d / v$ is the time since the source activity began, the hotspot of the receding jet is observed at a younger age given by:

$$
t_{\mathrm{rec}}=\left(\frac{1-\beta_{\mathrm{HS}} \cos \theta}{1+\beta_{\mathrm{HS}} \cos \theta}\right) t_{\mathrm{app}} .
$$

Therefore, the difference in age between the observed jet extremities, which must of course be imaged at the same "telescope time", is

$$
\Delta t=\frac{2 \beta_{\mathrm{HS}} \cos \theta}{1+\beta_{\mathrm{HS}} \cos \theta} t_{\mathrm{app}}
$$

What we actually observe is not just dependent on the kinematics of light-travel time, but also on the evolution of the luminosity in the jet material. To illustrate this point we consider the simple case where the X-ray, ICCMB emission depends on the time since emission from the central source in a manner qualitatively illustrated in Fig. 3.

In this picture the relic jet/counterjet plasma is moving at speed $\beta_{\mathrm{pl}}$; this is very likely to be slower than the speed at which the current jet or hotspots move otherwise we would not see any relic plasma along the counterjet. When the cooling is sufficiently rapid, then the cooling timescale $t_{\text {cool }}<\Delta t$, and the leading edge of the forward jet will be observed to cool and fade before the far extremity of the counterjet is observed to cool. The ratio of intensities for approaching and receding jets is dependent on the flux density, $S_{\nu} \propto \nu^{-\alpha}$, with $\alpha \approx 0.7$. We consider the flux ratio between equal volumes of plasma at either extremity of the approaching and receding jets,

$$
\frac{S_{\mathrm{app}}}{S_{\mathrm{rec}}}=\left(\frac{1+\beta_{\mathrm{pl}} \cos \theta}{1-\beta_{\mathrm{pl}} \cos \theta}\right)^{3+\alpha} \frac{L_{\mathrm{app}}\left(t_{\mathrm{app}}\right)}{L_{\mathrm{rec}}\left(t_{\mathrm{rec}}\right)}
$$

where we have ignored factors $O\left(\beta_{\mathrm{pl}}^{2}\right)$.

Initially, when neither jet has started to fade, the forward jet will be more luminous on the basis of size relative to the receding jet and Doppler boosting. Then, as shown in Fig. 3, the forward jet will 
be observed to fade first and the flux ratio will be observed to decline, giving a much more prominent counterjet. We measure the flux ratio for the relic jet over the relic counterjet in the $2-10 \mathrm{keV}$ band to be less than 0.25 , as the upper limit to any jet emission is about 4 times smaller than the measured counterjet emission. From the total observed length of the radio source of $130 \mathrm{kpc}$, we calculate (assuming $\theta=$ $60^{\circ}$ ) that the light travel-time difference between the hotspots is $2 \times 10^{5}$ years. Therefore the cooling time needs to be $\lesssim 10^{5}$ years. Thus, provided that the intrinsic luminosity of X-ray ICCMB fades within a light-crossing time $\left(2 \times 10^{5}\right.$ years $)$, the system will always evolve to a state where the receding jet has a greater X-ray luminosity than the older, approaching counterpart.

\section{Conclusion}

We have analysed the X-ray counterjet revealed by the combined $200 \mathrm{ks}$ Chandra ACIS-I image of Cygnus A. Its power-law spectrum, with photon index of 1.7, as well as the upper limit to the thermal electron density determined from radio polarimetry indicates that the feature cannot be explained by thermal gas. It is therefore likely to be emission from jet plasma having spectral index 0.7. Comparing the $\mathrm{X}$-ray detected counterjet with the observed radio counterjet in the $5-\mathrm{GHz}$ and $15-\mathrm{GHz}$ radio images, we conclude that the counterjet detected in X-rays is a relic jet. This conclusion was reached from the following observations: (i) the curvature of the outer parts of the X-ray counterjet is significantly different from that of the current radio counterjet; (ii) this feature lacks any directly associated radio emission implying a lack of high energy synchrotron particles; and (iii) the width of the X-ray counterjet is significantly broader than the radio jet or counterjet implying expansion.

\section{Acknowledgments}

The authors would like to thank St John's College, Oxford; the Royal Society and the Royal Irish Academy.

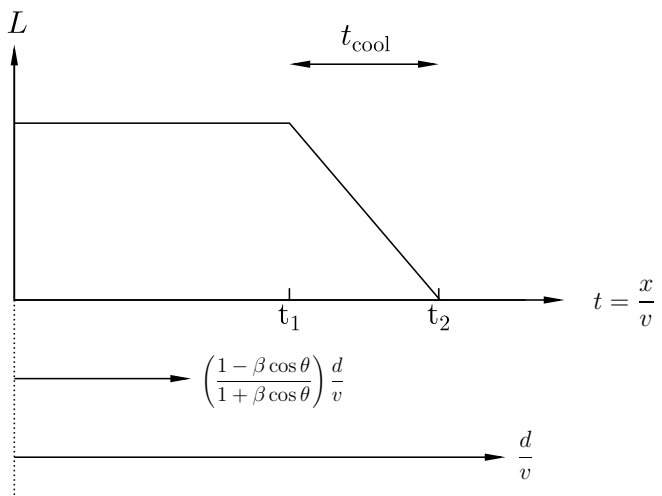

\begin{tabular}{|c|c|c|c|}
\hline app & on & fading & off \\
\hline on & & poss & obs \\
\hline fading & & poss & poss \\
\hline off & & & \\
\hline
\end{tabular}

Fig. 3. Upper: Schematic lightcurve for a fading jet. Lower: Illustrates the possible combinations for the fading jet and counterjet. The black squares are not formally possible, because of light-travel time effects while those in grey are formally possible but excluded by observation. The square labelled "obs" is the case we study in the text.

\section{References}

1. D. E. Harris and J. E. Grindlay, MNRAS 188, 25(July 1979).

2. M. C. Erlund, A. C. Fabian, K. M. Blundell, A. Celotti and C. S. Crawford, MNRAS 371, 29(September 2006).

3. K. M. Blundell, A. C. Fabian, C. S. Crawford, M. C. Erlund and A. Celotti, ApJL 644, L13(June 2006).

4. F. N. Owen, M. J. Ledlow, G. E. Morrison and J. M. Hill, ApJL 488, L15+ (1997).

5. J. S. Kaastra, R. Mewe and A. J. J. Raassen, Proc. Symp. New Visions of the X-ray Universe in the XMM-Newton and Chandra era (2002b).

6. K. C. Steenbrugge and K. M. Blundell, Ap\&SSS 310, 321(August 2007).

7. J. M. Dickey and F. J. Lockman, ARAA 28, 215 (1990).

8. A. S. Wilson, D. A. Smith and A. J. Young, ApJL 644, L9(June 2006).

9. R. S. Sutherland and G. V. Bicknell, Ap\&SSS, 325(August 2007).

10. R. S. Sutherland and G. V. Bicknell, ArXiv e-prints 707(July 2007).

11. J. W. Dreher, C. L. Carilli and R. A. Perley, ApJ 316, 611(May 1987).

12. A. H. Bridle and R. A. Perley, ARAA 22, 319 (1984). 